\title{
Multimodal Biometrics for Person Authentication using Hand Image
}

\author{
S.Sumathi \\ Research Scholar, Department of ECE, \\ Sathyabama University \\ Chennai-96, India.
}

\author{
R.RaniHema Malini. \\ Professor\& Head, Department of ECE, \\ St.Peter's College of Engineering \& Technology \\ Chennai-54, India.
}

\begin{abstract}
To obtain secure and accurate authentication system using most accepted biometrics Hand geometry in multimodal approach. Implement multi modal person authentication system using single input image with efficient features. In this proposed method Hand geometry and selected window size palm print features computed from same image is used for authentication. Integrating the features increases robustness of the system. Discrete Wavelet Transform (DWT) is used for feature extraction and Support Vector Machine (SVM) is proposed for classification. The final decision is made by fusion at matching score level. Experiments are carried out on the publicly available GPDS Hand Database. The achieved experimental results $\mathrm{GAR}=99.47 \%$, FAR $=0 \%$ showed the effectiveness of system considered for high security applications.
\end{abstract}

Keywords- Hand geometry, palm print, multi modal biometrics, DWT, SVM, Score level fusion.

\section{INTRODUCTION}

The rapid growth in the use of e-commerce, computer network login, national ID card, criminal investigation, reliable and secure access control is must for user identification. Every one has multiple accounts and passwords on an ever-increasing online transaction. Maintaining and managing access while protecting both the user's identity and the computer's data and systems has become increasing difficult. Every where security is the concept of authentication - verifying that the user is who he claims to be. Biometrics be the most secure and convenient method to satisfy the need for identity recognition of individual in the society. For automatic identification of an individual, physiological or behavioral characteristics of person are used [1].

Many types of single modal biometric systems have been developed, for example fingerprint, face, speech, palm print and hand geometry verification systems.To improve the performance of various biometric recognition systems by using better feature representation techniques and matching algorithms. Multibiometrics is another technique to improve the biometric recognition performance by combining multiple biometric traits. It aims to effectively fuse the salient information among the individual biometric traits which translates into better recognition performance. Single biometric systems are only capable to provide low to middle range of security feature. Thus for higher security, multimodal biometrics system is required. In addition, the industry is currently exploring the characteristics of multimodal biometric that are reliable, able to provide high security features, non-intrusive and widely accepted by the public [1].
Multimodal system also provides anti-spooling measures by making it difficult for an intruder to spool multiple biometric traits simultaneously. Due to its promising applications as well as the theoretical challenges, multimodal biometric has drawn more and more attention in recent years. Fusion of Hand geometry and palm print is user friendly and well accepted system with better performance. Feasibility and advantage over unimodal system and future direction of biometrics system are discussed in [2]. Several types of biometric features can be extracted from hand images: (i) hand geometry features, such as hand shape, palm area, width and length of fingers and other measurements; (ii) palm print characteristics, like principal lines, wrinkles, feature points, and skin texture.

The hand modality has a number of advantages in that it is user-friendly, hand-imaging devices are not intrusive, template storage costs are small and imaging conditions are less complex, relatively simple digital camera or flatbed scanner would suffice[3] Several systems have been developed using the hand modality. Two kinds of biometric indicators can be extracted from the low-resolution1 hand images; (i) palm print features, which are composed of principal lines, wrinkles, minutiae, delta points, etc., and (ii) hand geometry features which include area/size of palm and length and width of fingers.

Palm print based personal identification is a new biometric modality which is getting wide acceptance and has all the necessary traits to make it a part of our daily life. Palm prints are potentially a good choice for biometric applications because they're invariant with a person, easy to capture, and difficult to duplicate. They offer greater security than fingerprints because palm veins are more complex than finger veins. Compared with the fingerprint, the palm provides a larger surface area so that more features can be extracted. Palm print based personal verification has become an increasingly active research topic over the years.

Nowadays, wavelets have been used quite frequently in image processing. They have been used for feature extraction, denoising, compression, face recognition, and image superresolution. Wavelet transformations are a method of representing signals across space and frequency [4]. The decomposition of images into different frequency ranges permits the isolation of the frequency components introduced by "intrinsic deformations" or "extrinsic factors" into certain sub-bands. This process results in isolating small changes in an image mainly in high frequency sub-band images. Hence, discrete wavelet transform (DWT) is a suitable tool to extract the feature vector of a palm image.

Support vector machines (SVMs) are a set of related supervised learning methods that analyze data and recognize 
patterns, used for classification and regression analysis. A classification task usually involves with training and testing data which consists of some data instances. Each instance in the training set contains one "target value" (class labels) and several "attributes" (features). SVM has an extra advantage of automatic model selection in the sense that both the optimal number and locations of the basis functions are automatically obtained during training. [5, 6] propose wavelet function as a kernel function, the performance of SVM largely depends on the kernel. A SVM classifier can predict or classify input data belonging to two distinct classes. However, SVMs can be used as multiclass classifiers by treating a K-class classification problem as $\mathrm{K}$ two-class problems. This is known as one vs. rest or one vs. all classification.

The SVM classifier implementation is a standard one. In the MATLAB environment the LIBSVM software is used for processing. It is integrated software for support vector classification, regression and distribution estimation. It supports multi-class classification. The proposed system consists of three phases: (i) Hand geometry Recognition,(ii)Palm Print Recognition and (iii)Multimodal biometrics Fusion method.

Rest of the paper is organized as follows: Section 2 gives literature overview; section 3 gives methodology such as it briefly describes the unimodal hand geometry and palm print system followed by score level fusion. Section 4 shows experimental results and section 5 concludes the proposed work and future enhancement.

\section{RELATED WORK}

Jain et al. [7] described a verification system based on 16 hand geometrical features. The system was trained and tested using 500 images from 50 users. Sanches-Reillo et at. [8] Defined a scheme based on 31 hand-geometry features. The system was trained and tested using a database of 200 images of 20 users with $97 \%$ of success in GMM. Jain et al. [9] presented an authentication method based on the deformable matching of hand shapes. The system was tested on a database consisting of 353 gray-scale hand images (resolution $480 \mathrm{x}$ 485 ) of 53 people. The best results gave is $2 \%$ FAR and $3.5 \%$ FRR. Wong and Shi [10] proposed a peg-free hand geometry authentication system using a flatbed optical scanner for hand image capturing. The hand sizes and shape of the fingertip regions were measured. They achieved a genuine acceptance rate of $88.9 \%$ and a false acceptance rate (FAR) of $2.2 \%$ with 30 hand features. Bulatov et al. [11] measured 30 geometric sizes on a hand image. Circles were fitted into different areas of fingers and the palm. The radiuses, perimeters, and areas of the circles, together with the lengths and widths of fingers, were measured. They achieved an FAR of $1 \%$ and a false rejection rate (FRR) of $3 \%$ for verification. Boreki et al. [12] profiled curvatures along the hand contour and found landmark points to separate each finger. An FAR of $0.8 \%$ and FRR of 3.8\% were reported based on 360 images of 80 users. Palm print Recognition system use prominent palm-line features, texture, global texture energy, or combination of these characteristics discussed in [13]. A frequency domain feature extraction algorithm for palm-print recognition is proposed [14], which efficiently exploits the local spatial variations in a palm print image based on extracting dominant spectral features from each of these bands using two dimensional discrete cosine transform (2D-DCT). A scannerbased personal authentication system by using the palm print features is proposed in [15]. A new method to authenticate individuals based on palm print identification and verification is described in [16] via multiple feature extraction. A recent paper by Han et al. [17] uses morphological and Sobel edge features to characterize palm prints and trained a neural network classifier for their verification.

Kumar et al [18] proposed the palm print and hand geometry features of an individual are obtained from the same hand image. Two schemes for the fusion of features, fusion at the decision level and at the representation level, were considered. The decision level fusion with max rule gave better results. In [19] Ribaric proposes the combination of eigen palm and eigen fingers at the score level. The database consists of 5 templates of 110 users claiming a $0 \%$ EER in verification and $0.58 \%$ of Error at identification. The paper by A. Kumar et al [20] describes improvements to the performance of a palm print based verification system by integrating hand-.geometry and palm print features. A digital camera (1280 x 960 pixels) is used to acquire the hand images. The Fusions at the featuregeneration level as well as at the decision level were checked. Experimental results, on the Image dataset of 100 users, show that the combination at decision level gives the best results. Ong et al [21] proposed fusion method by using weighted sum rule and SVM with RBF kernel for palm print and hand geometry features. [22] Discussed Hand geometry, palm print and finger surface biometric features are used for fusion. Two different levels of fusion are applied for authentication such as score level fusion for the five finger surface features and decision level fusion for various modalities, based on the majority vote rule. The recognition of individual using average half face and selective palm print based on DWT and SVM classifier with score level fusion discussed in [23].

Previously, Euclidian distance, Mahabaloni's distance, nearest neighbor $(\mathrm{NN})$, the probabilistic decision-based neural network, hidden markov model [24 25] be used as a classifier. Based on the better performance, we combine Discrete Wavelet Transform, city block distance classifier and SVM fusion at score level. Hence it is proposed to implement with the new algorithm.

\section{METHODOLOGY}

The proposed system combines two biometric modalities, namely hand geometry and palm print. The hand geometry and

palm print features are extracted using the same hand image.

\subsection{Hand geometry}

The hand contour is obtained from monochrome hand image. Trace the boundaries and give coordinates of the starting points, then calculate the distance from wrist centre to contour distance. The radial distance represents local maxima and minima. The peaks of the radius function locate the finger ends and the minimums of the radius indicate the valleys between fingers. This procedure is summarized in Fig.1 [26]. The hand geometry features such as length and width of fingers, thickness from the hand image. A total of 16 hand geometry features were used (Fig.2); 5 finger lengths, 10 finger widths ( 2 widths per finger) and palm width. Genuine and imposter scores are generated using city block distance. 


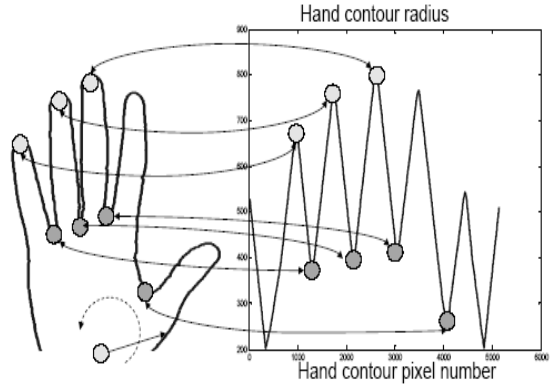

Fig 1: Hand contour and radius. The ends and valleys between finger corresponds to the maximum and minimum of the radius

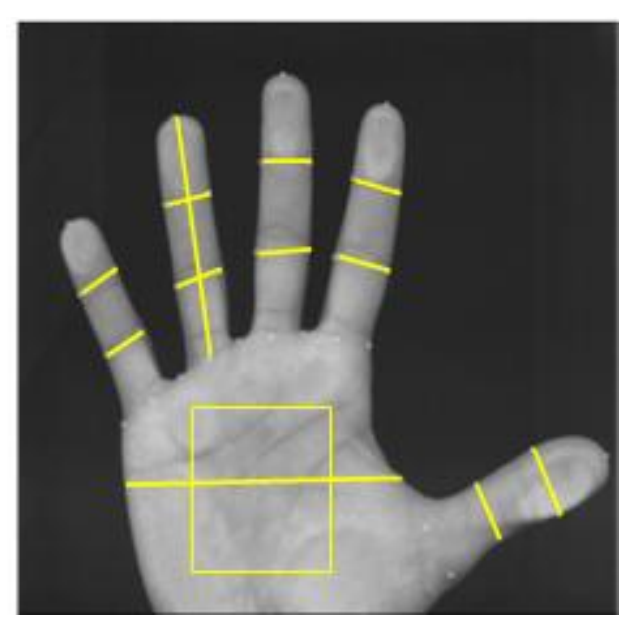

Fig 2: Hand geometry features

\subsection{Palm print}

The feature extraction stage is shown in Figure 3. First, the given palm print image is decomposed by using Haar wavelet transform. From 3-Level wavelet decomposition, 10 subbands are obtained. The selection of wavelet coefficients as features depends on the size of the window placed over the center of each sub-bands as shown in Figure 4. The wavelet coefficients for palm print identification are reduced by using specified window size. Experiments are conducted for various window sizes and the best window size of $60 \%$ which gives better success rate is chose.

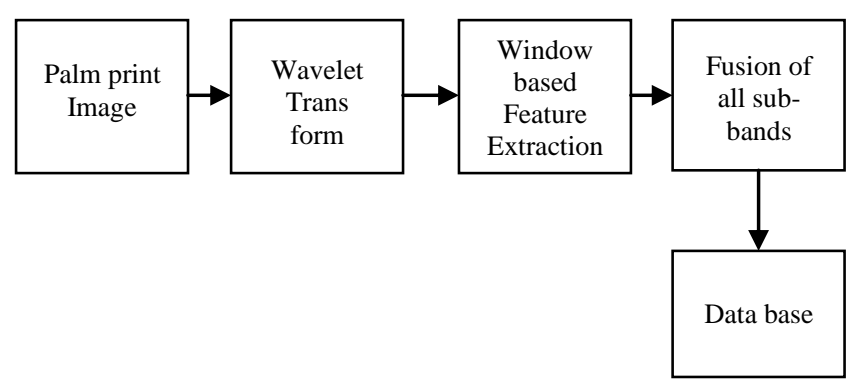

Fig 3: Feature Extraction of palm print

The selected features from the 10 sub-bands are fused together in a serial manner starting from the detailed coefficients to approximate wavelet coefficients. Performance measures of selective window size palm features tested with Hong Kong Multi spectral palm print data base and IIT palm print data base discussed in [27 a \& b].City block distance be used to generate genuine and imposter scores.

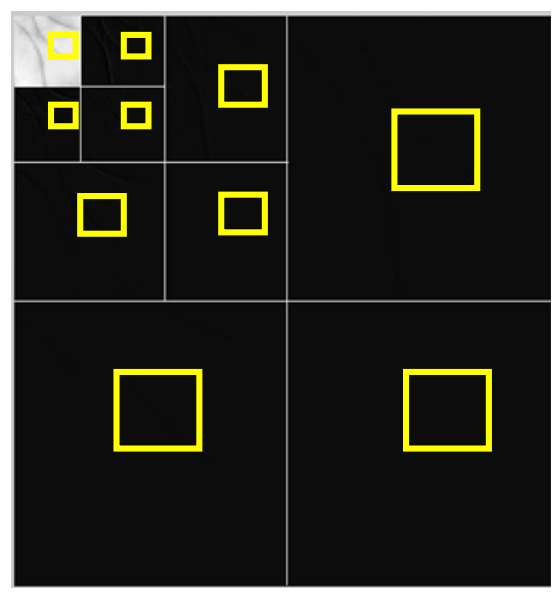

Fig 4: Selection of Wavelet coefficients in PIP

Normalization is essential for assigning two different modalities in the same range. The generated scores of Hand geometry and palm print are normalized using z-score before fusion. This is normally essential for the raw features getting from different modalities can be merged in the fusion stage. The final matching score is applied to SVM based fusion to recognize the person as genuine or an impostor. 


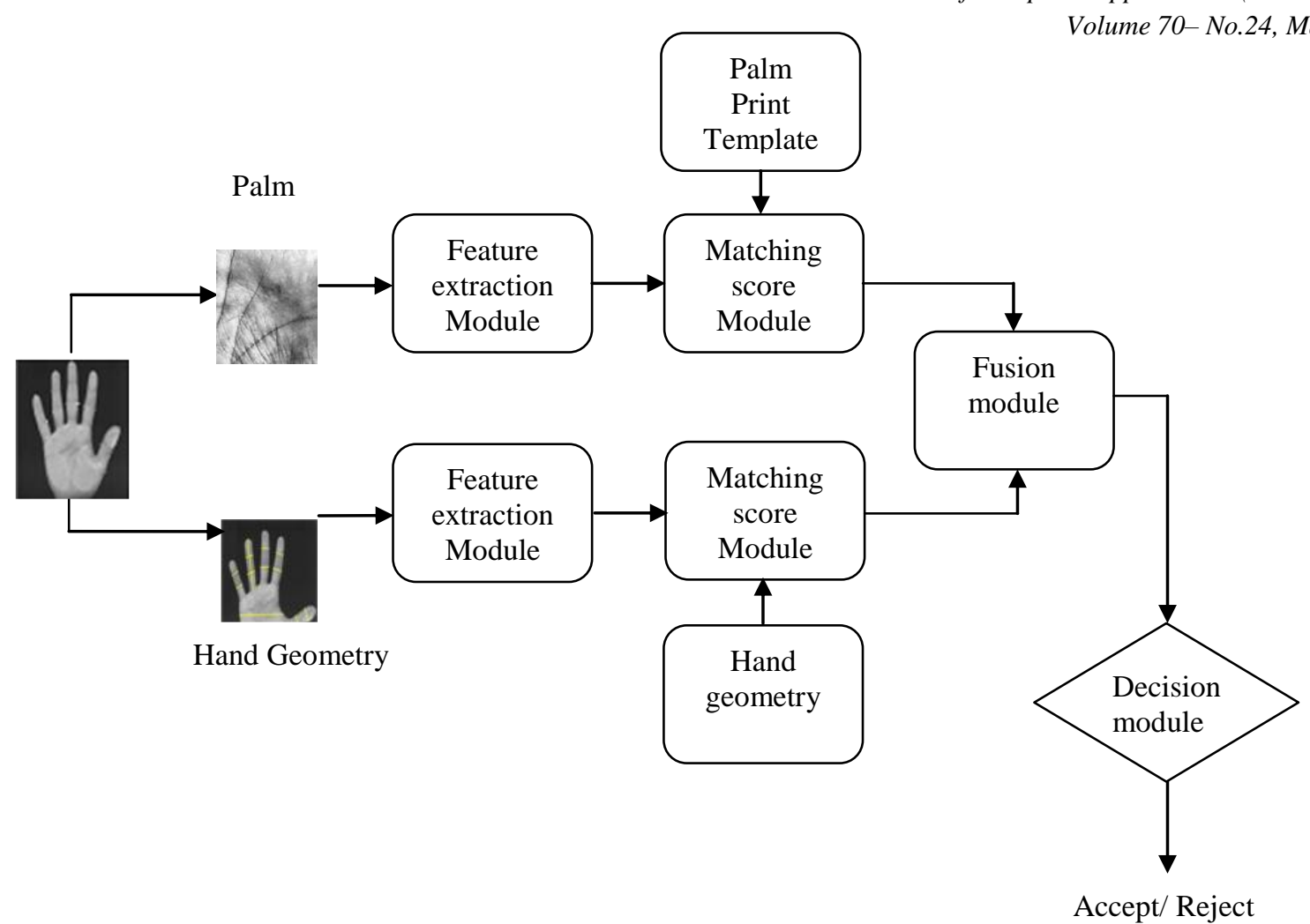

Fig 5: Block diagram of hand geometry and palm print multimodal Biometric system

\subsection{Multimodal biometrics Fusion method}

One or more biometric traits combine to provide new independent information that gives the opportunity to greatly improve recognition performance. The fusion of two modalities allows reducing the impact of system failure than unimodal biometrics. Multimodal biometrics has many advantages, such

as providing greater universality to the system, to over come spoof attacks. The multi biometric system includes four principal modules: the sensor module, the feature extraction module, the matching module, where parameters are compared to the template database, and the decision module. Information fusion can occur in any of the aforementioned modules: data normalization followed by a concatenation of the biometric data vectors in pixel or feature fusion [28]; combination of the matching scores to produce a single new score in score fusion [29]; combining the decisions of the different classifiers according to certain rules to give the final decision in decision fusion [30].

In our multimodal biometric system, the fusion is performed at the matching score level. In general score level fusion method can be divided into three [31 32] i.e. transformation based score level fusion (eg sum rule based fusion), classifier -based score level fusion(eg SVM based fusion), density based score level fusion(eg likelihood ratio test with Gaussian Mixture model). In this proposed method use classifier based score level fusion i.e., support vector machine (SVM)-based fusion.

The main concept of SVM is to separate the training data into two classes with a hyper plane that maximizes the margin between them.[33] Considering a two-class classification to be

solved by a SVM, we start with a training sample described by a set of features $x i \in R n$; $n$ being the number of features, belonging to one of the two classes designated by the label
$\mathrm{Yi} \in\{+1,-1\}$. Yi is set to 1 for genuine vector score and -1 for imposter vector score. After performing the training phase, one can determine whether a testing vector score xi belongs to a genuine class or imposter class.

More than one biometric system can be integrated to improve the performance of the recognition system. Treat the matching scores of different modalities as a feature vector and use SVM based fusion to classify the vector as being genuine or an impostor. Given the query image as input, features are extracted. Combining the matching scores of two traits i.e. hand geometry and selective window size palm print. The final matching score is applied to SVM based fusion to recognize the person as genuine or an impostor. The multimodal biometric system is developed using two traits (hand geometry and selective palm print) is shown in fig 5 .

\section{EXPERIMENTAL RESULTS}

The experiment of the proposed system consists in the evaluation of hand geometry, selective window size palm print and fusion of hand geometry and selective window size palm print in score level. GPDS database [34] is used to execute the proposed method. It consists of 10 different acquisitions of 150 people by a desk scanner. The 1500 images have been taken from the users' right hand. The experiments are conducted for 45 subjects randomly chosen from the database. From each subject 5 different acquisitions used in the Training phase and the remaining 5 are used in the Testing phase. The table shows the result for three distinct cases, (i) hand geometry alone, (ii) palm print alone, and (iii) score level fusion of hand geometry and palm print

Table.1 GAR and FAR for single modal and multi modal

\begin{tabular}{|c|c|c|}
\hline & GAR \% & FAR \% \\
\hline Hand geometry & 92.70 & 0.000357 \\
\hline Selective Palm & 97.91 & 0.000894 \\
\hline
\end{tabular}




\begin{tabular}{|c|c|c|}
\hline $\begin{array}{c}\text { Score Level } \\
\text { Fusion }\end{array}$ & 99.47 & 0 \\
\hline
\end{tabular}

To assess the performance of the proposed system, many computer simulations and experiments with GPDS database are performed. The performance measure Genuine Acceptance Rate (GAR) and False Acceptance Rate (FAR) are calculated. The system is implemented in MATLAB version 7.6. Table 1 clearly shows the benefit of multimodal.

\section{CONCLUSION}

In this paper, we have proposed a new approach for biometric authentication that is based on hand geometry .Users can place their hands freely on the scanner surface without need of pegs to fix the placement of the hand. The features used for matching are the fingers length, width, palm width and palm features. The objective of this work was to investigate the integration of palm print and hand geometry features, and to achieve a better performance that may not be achievable with single biometric indicator alone.

Compared to the literature, proposed system is able to achieve similar and better performance to other hand based multimodal systems [18 19]. In ref [19] a max recognition rate of $99.28 \%$ is achieved by fusion of palm print and finger surface features. Another multimodal system [18] achieved a max recognition rate of $98.59 \%$ and $0 \%$ FAR by fusion of palm print and hand geometry features. Using finger surface, palm print and hand geometry features with decision level fusion [22], max recognition rate of $98.28 \%$ and FAR $0.31 \%$ were also discussed.

\section{REFERENCES}

[1] A.K. Jain, A., Ross, \& S. Prabhakar, "An Introduction to biometric recognition," IEEE Transactions on Circuits System and Video Technology, vol. 14 (1), pp. 4-20, 2004.

[2] Ashish Mishra, 'Multimoda Biometrics it is; Need for Future systems', International Journal of Computer Applications, vol 3-No 4,June 2010,pp 28-33.

[3] Hand-based Biometrics, Biometric Technology Today, vol. 11, no. 7, 2003, pp. 9-11.

[4] R.M.Rao and A.S.Bopardikar, "Wavelet TransformsIntroduction to theory and Applications", Addison Wesley Longman,1998.

[5] C.J.C.Burges, "A Tutorial on support vector machines for pattern Recognition", Data Mining and Knowledge discovery,vol 2:121-167,1998.

[6] M.Pontil, A. Verri, "Support Vector Machines for 3-d Object Recognition", IEEE Transactions on Pattern Analysis and Machine Intelligence, pp. 35-39, 1998.

[7] A. K. Jain, A. Ross and S. Pankanti, uA Prototype Hand

Geometry-based Verification System", 2nd Int. Conference on Audio- and Video-based Pernal Authentication (AVBPA), Washington, pp. 166-171, March 1999.

[8] R. Sanchez-Reillo, C. Sanchez-Avila and A. GonzalezMarcos, "Biometric Identification Through Hand Geometry Measurements", IEEE Transactions onPaffem
The experimental results showed the effectiveness of system in terms of GAR and FAR. With hand geometry $92.70 \%$ and $0.000357 \%$, with selected palm print feature $97.91 \%$ and $0.000894 \%$ and with fusion of hand geometry and palm print $99.77 \%$ and 0 .

In the future work we plan to combine these modalities with decision level fusion with different classifiers. Propose multi biometrics system with serial and parallel fusion of uncorrelated modalities. Fig 7 shows the GUI of multi modal recognition.

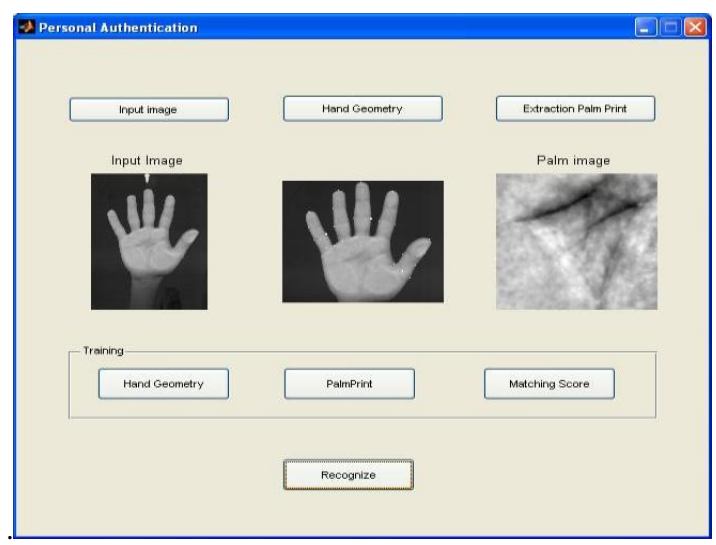

Fig 6: GUI of multimodal Recognition

Analysis Machine Intelligence, Vol. 22, No. 10, pp. 11681171,2000

[9] A. K. Jain and N. Duta, "Deformable Matching of Hand Shapes for Verification',Proceedings of IEEE International Conference on Image Processing, Kobe, October 1999.

[10] A.Wong and P. Shi, "Peg-free hand geometry recognition using hierarchical geometry and shape matching," in Proc. IAPR Workshop on Machine Vision Applications, Nara, Japan, Dec. 2002, pp. 281-284 [Online]. Available:

http://www.ee.ust.hk/ eeship/Papers/MVA02.pdf.

[11] Y. Bulatov, S. Jambawalikar, P. Kumar, and S. Sethia, "Hand recognition using geometric classifiers," in Proc. 1st Int. Conf. Biometric Authentication, Hong Kong, China, Jul. 2004, pp. 753-759.

[12] G. Boreki and A. Zimmer, "Hand geometry: A new approach for feature extraction," in Proc. 4th IEEE Workshop on Automatic Identification Advanced Technologies, Oct. 2005, pp. 149-154.

[13] D. Zhang, W.K. Kong, J. You, and M. Wong, "Online Palm Print Identification," IEEE Trans Pattern Analysis and Machine Intelligence, vol. 25, no. 2, pp. 1041-1050, Feb. 2003.

[14] Hafiz Imtiaz and Shaikh Anowarul Fattah, "A DCTbased Feature Extraction Algorithm for Palm-print Recognition", IEEE International Conference on Communication Control and Computing Technologies, October 2010, pp 657-660. 
[15] Chin-Chuan Han and Hsu-Liang Cheng, "Personal Authentication Using Palm Print Features", 5th Asian Conference on Computer Vision, January 2002, pp 1-6.

[16] Jane You and Wenxin Li, "Hierarchical palm print identification via multiple feature extraction", Journal of Pattern Recognition, April 2002, pp 847-859.

[17] C.C. Han, H.-L. Cheng, C.-L. Lin and K.-C. Fan, "Personal authentication using palm print features," Pattern Recognition, vol. 36, pp. 371-381, 2003.

[18] Kumar, A., Wong, D., Shen, H. and Jain, A., "Personal Verification Using Palm print and Hand Geometry Biometric", in Proc. 4th International Conference on Audio- and Video-based Biometric Person Authentication, Guildford, U. K., June 2003, pp. 668-678.

[19] S. Ribaric, 1. Fratric, "A Biometric Identification System Based on EigenPalm and EigenFinger Features", in IEEE Transactions on Paffem Analysis and Machine Intelligence", vol. 27, no. 11, pp. 1698-1709, November 2005.

[20] A. Kumar, D.Zhang, "Personal Recognition Using Hand Shape and Texture", in IEEE Transaction on image Processing, vol. 15, no. 8, pp. 2454-2461, 2006.

[21] M. Ong, T. Connie, A. Jin, and D. Ling, "A single-sensor hand geometry and plamprint verification system," in Proc. ACM SIGMM Workshop Biometrics Methods and Applications, Berkley, CA, 2003, pp. 100-106.

[22] T Sanches, J Antunes, and P L Correia, "A Single Sensor Hand Biometric Multimodal System," in 15th European Signal Processing Conference (EUSIPCO), Poznan, Poland, 2007, pp. 30-34

[23] S.Sumathi and R.Ranihemamalini, "Multi-biometric Authentication using DWT and Score Level Fusion", European Journal of Scientific Research, Vol 80, No 2 pp 213-223,2012.

[24] J. Kittler, M. Hatef, R. Duin, and J. Matas, "On combining classifiers," IEEE Trans. Pattern Anal. Mach. Intell., vol. 20, no. 3, pp. 226-239, Mar. 1998.

[25] S.H.Lin ,S.Y.Kung and L.J.Lin,"Face Recognition / Detection by Probalistic Decision -based Neural Network",IEEE Trans.Neural Networks,vol 8,no 1,pp 114-132,Jan.1997.
[26] Miguel A. Ferrer1, Joan Fàbregas2, Marcos Faundez2, Jesús B. Alonso1, Carlos Travies "Hand Geometry Identification System Performance" IEEE conference2009,pp167-171.

[27a] S.Sumathi and R.Ranihemamalini, "Personal Identification Using Palm Print Features With an Efficient Method of DWT" Springer,Part II, CCIS 270,2012,pp337-343

[27b] S.Sumathi and R.Ranihemamalini, "An Efficient Method for Person Identification Using Wavelet Transform with Palm Print Features", National Journal on Electronic science and Systems (NJESS), 2011. Vol 2/pp51-56/No 1 ISSN No: 0975-7325

[28] X.-Y. Jing, Y.-F. Yao, D. Zhang, J.-Y. Yang, and M. $\mathrm{Li}$,"Face and palmprint pixel level fusion and kernel dcv-rbf classifier for small sample biometric recognition," Pattern Recognition, vol. 40, no. 11, pp 3209-3224, 2007.

[29] L. Hong, A. Jain, and S. Pankanti, "Can multibiometrics improve performance," in Proceedings of IEEE Workshop on Automatic Identification Advanced Technologies, pp. 59-64, 1999.

[30] L. Xu, A. Krzyzak, and C. Y. Suen, "Methods of combining multiple classifiers and their applications to handwriting recognition," IEEE Transactions on Systems, Man and Cybernetics, vol. 22, no. 3, pp. 418-435, 1992.

[31] K. Nandakumar, Y. Chen, S.C. Dass, A.K. Jain, Likelihood ratio-based biometric score fusion, IEEE Transactions on Pattern Analysis and Machine Intelligence 30 (2) (2008) 342-347.

[32] C. Bergamini, et al., Combining different biometric traits with one-class classification, Signal Processing 89 (11) (2009) pp 2117-2127.

[33] M. He, et al., Performance evaluation of score level fusion in multimodal biometric systems Pattern Recognition (2009), doi:10.1016/j.patcog.2009.11.018

[34] GPDS hand database, http://www.gpds.ulpgc.es/download/. 